\title{
Métodos y técnicas para la recuperación del registro arqueológico: una mirada desde el presente
}

\author{
Xavier Roda Gilabert, Rafael Mora Torcal ${ }^{1}$
}

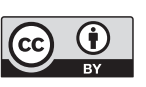

Recibido: 18-10-2014

Aceptado: 10-11-2014

\section{Resumen}

La renovación metodológica que ha surgido en los últimos años en el ámbito de la arqueología prehistórica ha favorecido la aparición de nuevas técnicas y estrategias de excavación que facilitan una mejor comprensión del registro arqueológico. Este volumen recopila una serie de textos que presentan diferentes aspectos metodológicos, cuyo objetivo es servir como introducción de los diferentes técnicas abordadas: geoarqueología, fitolitos, escáner 3D. El recorrido discurre desde la prospección arqueológica hasta las sistemáticas de excavación, repasando las diferentes metodologías que contribuyen a una mejor contextualización y preservación de los restos recuperados.

Palabras clave: trabajo de campo; sistemas de registro; metodología; muestreo; aplicaciones informáticas; arqueografía.

Resum. Mètodes i tècniques per a la recuperació del registre arqueològic: una mirada des del present

La renovació metodològica que ha sofert l'arqueologia en els darrers anys ha afavorit l'aparició de noves tècniques i metodologies d'excavació que faciliten una millor gestió del registre arqueològic. Aquest volum recull una sèrie de textos que presenten diversos aspectes metodològics i tenen com a objectiu servir d'introducció a les diverses tècniques abordades: geoarqueologia, fitolits, escàner $3 \mathrm{D}$. El recorregut va de la prospecció arqueològica a les tècniques d'excavació, repassant les diverses metodologies que contribueixen a una millor contextualització i preservació del registre arqueològic.

Paraules clau: treball de camp; sistemes de registre; metodologia; mostratge; aplicacions informàtiques; arqueografia.

Abstract. Methods and techniques for recovering the archaeological record: a view from the present

In recent years has been emerged a renewal in archaeological methodology that encouraged the appearance of new techniques that facilitate fieldwork better management of the archaeological record. This volume collects a series of papers that present different methodological aspects and

1. Centre d'Estudis del Patrimoni Arqueològic de la Prehistòria (CEPAP-UAB). Universitat Autònoma de Barcelona. Facultat de Lletres. 08193 Bellaterra (Cerdanyola del Vallès).

javier.roda@uab.cat; rafael.mora@uab.cat 
aimed to serve as an introduction to the addressed themes: Geoarchaeology, phytoliths, geological factories, Data matrix codes; 3D scanner. Thus the texts ranging from archaeological survey to the excavation techniques reviewing the different methodologies that contributes to a better contextualization and preservation of the archeological record.

Keywords: Fieldwork recording systems; methodology; sampling; Computer applications; Archaeography.

Roda Gilabert, Xavier; Mora Torcal, Rafael. «Métodos y técnicas para la recuperación del registro arqueológico: una mirada desde el presente». Treballs d'Arqueologia, 2014, núm. 20, p. 3-5. DOI: $10.5565 / \mathrm{rev} / \mathrm{tda} .54$

A lo largo de los últimos 25 años, la investigación arqueológica ha experimentado profundos cambios metodológicos y técnicos que han afectado especialmente a las actividades relacionadas con el trabajo de campo y la recuperación del registro fósil.

La aplicación de las tecnologías de la información y la comunicación en la sistemática de la recuperación del registro arqueológico, así como el desarrollo de la microinformática, han revolucionado completamente el procedimiento. Los actuales equipos no solo son capaces de procesar y gestionar bases de datos, sino que además pueden visualizarse, analizarse y entrecruzarse los distintos tipos de datos.

En el ámbito metodológico, nuestra perspectiva pasa por poner de relieve el significado de los ítems arqueológicos a partir de su contextualización. Ello implica considerar la recuperación del registro arqueológico como una parte relevante dentro del proceso de investigación y que está estrechamente relacionada con su interpretación. Esta noción de recuperación contextual es un compromiso ético dirigido a que esa información pueda ser reinterpretada en el futuro.
En nuestro país, el rol de las universidades en la creación, difusión y homogeneización de los métodos y las técnicas básicos en la recuperación del registro arqueológico ha sido prácticamente inexistente hasta hace pocos años, en que se ha iniciado la formación en el ámbito de las habilidades meto dológicas y técnicas de campo con la creación de grados especializados. Sin embargo, sigue ocupando un lugar secundario dentro de las enseñanzas académicas y se deja que sea el propio sujeto quien, a través de su propia iniciativa, genere su experiencia de campo. El resultado es una enorme disparidad de criterios y la poca renovación de los parámetros constructivos en esta área.

Por todo ello, el presente volumen se propone un doble objetivo: en primer lugar, dar a conocer las distintas estrategias desarrolladas para la sistematización de la recuperación del registro arqueológico, la contextualización y preservación del mismo; y en segundo lugar, presentar textos que sirvan como puerta de entrada a cada uno de los ámbitos de actuación que abordan. Paralelamente, queremos insistir en la necesidad de homogeneizar los sistemas de recogida de información y su posterior procesado. Aunque se han 

arqueológico: una mirada desde el presente

hecho intentos de sistematización - muchos de los cuales comparten una filosofía similar en el tratamiento de los datos arqueológicos-, no se ha podido consensuar un sistema unificado. A nuestro entender, la ausencia de un sistema común impide el intercambio de información entre proyectos y limita la posibilidad de comparar contextos y registro.

Los diferentes artículos abordan cuestiones que van desde ejemplos de protocolos metodológicos seguidos en la recogida de datos hasta estudios específicos realizados sobre determinados tipos de materiales. De este modo la metodología de excavación se presenta en dos textos (Mora et al.; De la Torre et al.). Paralelamente, Benito et al. sintetizan los conceptos básicos de la geoarqueología presentando los métodos de la geología, la geomorfología y la estratigrafía y cómo contribuyen estas ciencias a la reconstrucción de los paisajes y los procesos de formación de los yacimientos.

Estos últimos puntos son abordados de manera específica en dos de las contribuciones. La interpretación de los depósitos sedimentarios en los cuales se han conservado los restos arqueológicos desempeñan un papel clave en la interpre- tación de esta metodología, que actualmente se integra de forma habitual en la toma de datos en el trabajo de campo. En este sentido, el estudio de las fábricas arqueológicas (Roy et al.), es decir, la orientación y el ángulo de buzamiento de los objetos, se presenta como una técnica capaz de aportar datos sobre los procesos de formación de los yacimientos trabajados. Por su parte, el trabajo de Albert y Portillo presenta un estado de la cuestión sobre los estudio de los fitolitos, restos que se han convertido en indispensables en las reconstrucciones paleoambientales. Las investigadoras abordan cuestiones metodológicas y repasan los procesos postdeposicionales que pueden afectar a la preservación de este tipo de restos microvegetales.

Finalmente, se muestra la aplicación de los códigos Data Matrix (DM) en el trabajo de campo y la catalogación (Roda Gilabert et al.). Esta técnica se presenta como un método rápido y eficaz que es capaz de substituir el siglado tradicional con escasa inversión en infraestructuras. Gracias a esta nueva técnica, se disminuyen los errores, se facilita el almacenaje $y$, en definitiva, se ayuda a la preservación del patrimonio arqueológico. 
